\title{
99 NORMALISING ACP IN THE COMMUNITY
}

B Hammes, ${ }^{1}$ Linda A Briggs ${ }^{1}$ 'Gundersen Health System

10.1136/bmjspcare-2013-000491.99

Background Advance care planning is the process of coming to a greater understanding of future medical decisions; personally reflecting on those decisions; discussing the options and plans with others who are close; and creating a clear plan of action. This is a 
process that needs to be part not only of the health care system, but also of the community life.

Aim To explore the way that the process of advance care planning can become part of family and community life.

Discussion While advance care planning is often focused on medical treatment decisions, for example, resuscitation, in reality such planning is more about values and goals of individuals. As such, it has as much to do with fundamental beliefs, obligations; relationships; and values as it does about information regarding a treatment. It is coming to a fuller and timely understanding of these aspects of an individual's life that the goals of medical care and the treatment decisions get shaped. In this regard advance care planning is a human activity that involves relationships and activities like religion and culture. Such conversations belong as much in the community as they do in the hospital. This broader approach, however, is not well understood by the larger community nor is it fully trusted. To have a highly successful advance care planning approach requires the engagement of a wide variety of organisations and institutions from the community. This presentation will explore effective methods of such engagement and consider how it improves outcomes. 